\title{
Alphabetirare Sadjregifter.
}

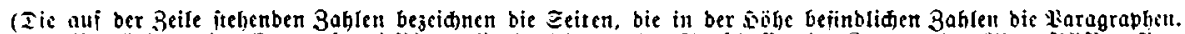

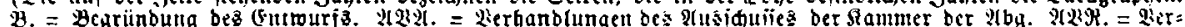

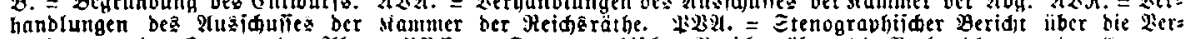

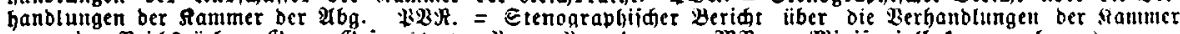

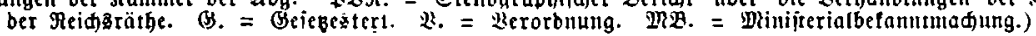

9.

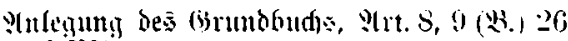
(ख)(B⿺辶)

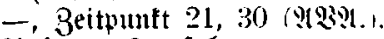

anlegungsoerfabren

(i. aud) Eintrayun a jolienfreier (Srund:

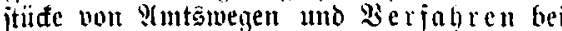
ber (Eintragung folienjieicr Grunditï(t)).

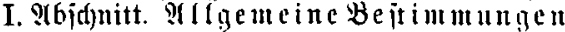
$166-168$ (M)

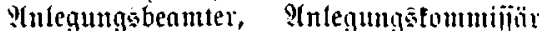
$157^{3}$ (23.).

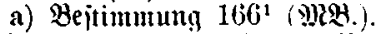

b) 3 uitündigteit $166^{2}$ (싼)

c) Sienitantritt $166^{3}(\mathfrak{M R B}$.).

d) 三dreibuerf $166^{+}$(MlQ

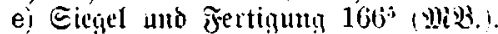

Nibeitsplan $\left.16^{\circ}, 9\right](8)$.)

Ufufid)reibGud) $167^{7}$ (Dis.).

Tngebuth $16^{\circ}$ (M) 3 .).

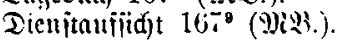

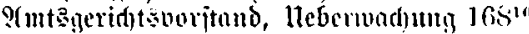
(y)is. $)$.

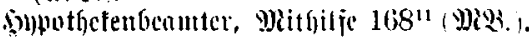

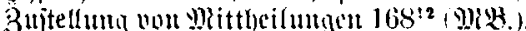

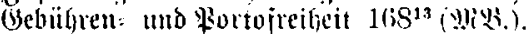

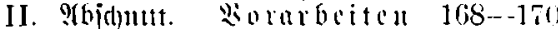
(ำ?

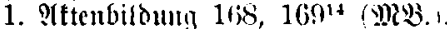

2. Tejtitellun ber jolienfreicu Brandoitiote 169 (ํ)?

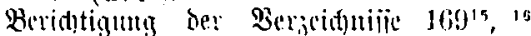
(i) $(2)$ B.).

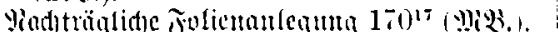

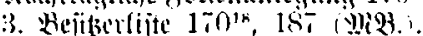

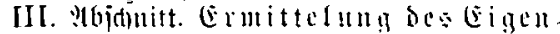

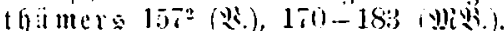

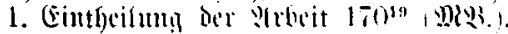

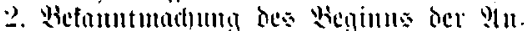

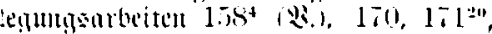
$18 \mathrm{~s}$, ind

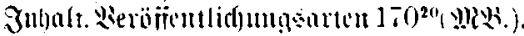

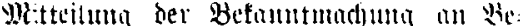
jörben $17^{21}$ (Wlive).

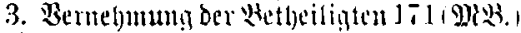
Betbeilinte $15 \mathrm{~S}^{5}$.

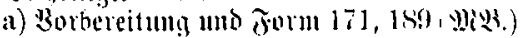

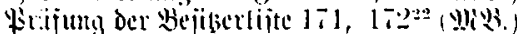

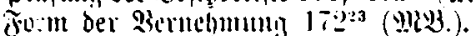

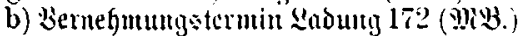

End de Termin: $172^{2+}$ (ำ?

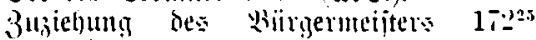

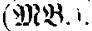

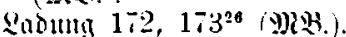

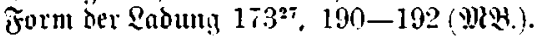

llmiang bes Termins $173^{28}$, why.).

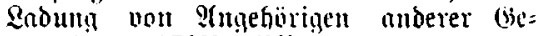
meinden $173^{29}$ (\$)(B.).

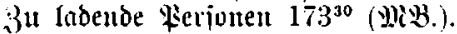

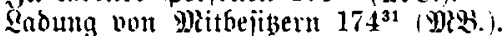

כुolgen und Etraje bes llngehoriants $158^{\circ}$

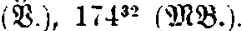

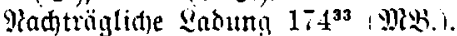

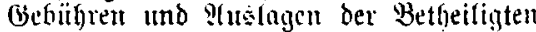
$17 t^{3+}$ (miB.).

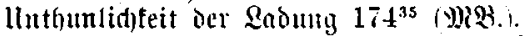

Bernehmung anierhalb bes 2 ermins $17 t^{36}$ (D)?

Bermehmung auswärtiger \&iscronen $17 t^{37}$ (\$2B.).

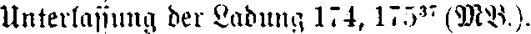

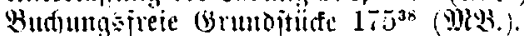

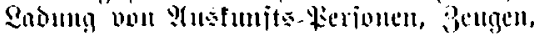
Eadiveritïndigen $175^{40}, 193$ (9)(3).

c) Vicrhandumpen äber bic Eig̣enthums:

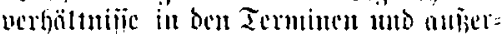

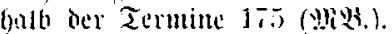

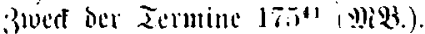

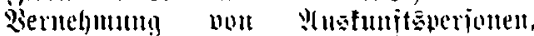

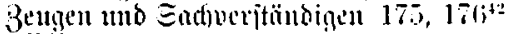

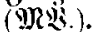

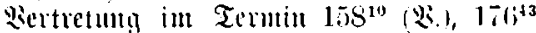
(1)!2s.).

(B) (Wliv.).

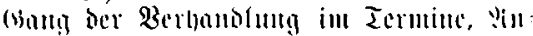
D)

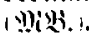

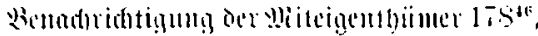

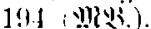

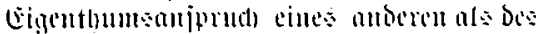
futaitermiojigen Weitgirs:

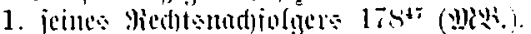

2. cinter anderent Weriont.

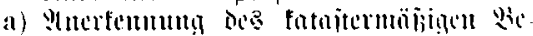

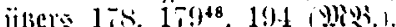

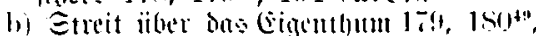
195,$196 ;(0)$ i. 3.1.

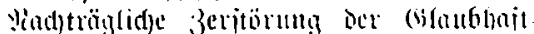

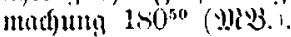

Midnterid)einten in Tommin $1800^{51}$ ( 19 \& 
Qnvronung ber Eintragung ohne $\mathfrak{B e r}=$ nehmung $180^{\text {52 }}$ (MRS.).

d) Brotofollirung der Berhanolungen 180 , 196 (DiSB.).

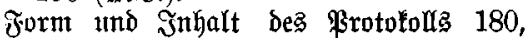
$181^{53}$ (MDS.).

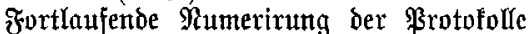
$181^{54}$ (MBS.).

Bertweifung auf bie Bejitzerlīte und bas

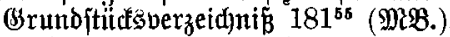

Bezeidnung ber vorgelegten llrtunten $181^{\text {s6 }}(\mathfrak{M P B}$.).

Unterfarreibung ber Erfflärungyen $118^{\text {b7 }}$ (MBO.).

Bermerf der Ânoronungen und Rit= theilungen $181^{\text {s8 }}$ (MMP.).

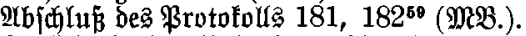

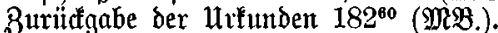

Bermerte in ber Bejiberlijte und im

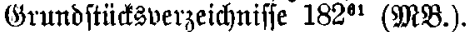

e) Beurfundung von Berträgen, Erflä= rungen unt Eintragung şbervilligungen 182 (פRS.)

3ujtünoigfteit $182^{\circ 2}(\mathfrak{M Q B}$.).

ऊnfalt und Borautsfewungen $182^{63}$ (MBB.). Furnt $182,183^{64}$ (MBS.).

syittheilung itber bie Beurtundungen an das Rentant $183^{65}$ (MBB.).

f) Anderyeite Ergebnifije der Erntittelungen,

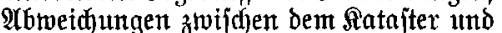
bem Bejizitant $183^{\circ 8}$ (MBB.).

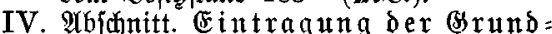
it îte und ihrer Eigenthit mer 183 fit 184 (MMR.).

Beitpunft ber (5intragung $183^{87}$ (MPB.)

Form und Jnhalt ber Eintragunt 183, $184^{68}$ (MDOS.)

PInleaunto ber Folten $184^{68}$ (MMB.).

Berifhtigung ber Regiter $184^{70}$ (MB.).

V. Mbfdritt. Berzeidnit ber nidit eingetragenen (Srunditín $160^{20}$ (B.), 184-185 (MBB.).

Fornt und Jnhalt 184, 185"1 197 (MMB.).

Unterjøreibung. Beriđtigung $185^{72}$ (MRB.).

VI. Mbjdnitt. Bejondere Red ţoer:

bältuiffe 185-186 (MBB.).

1. Stoftwerłgeigenthum $160^{17}\left(\mathfrak{B}\right.$.), $185^{73}$ (MRB.).

2. Realverbünde $185^{74}$ (MB.).

3. (Erbbauredite $160^{18}, 162^{28}(\mathfrak{B}),. 185^{75}$ (MNB.).

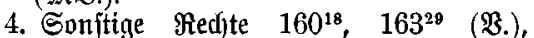
$185^{78}$ (DiB.).

5. Bergwerk?eigenthum $162^{28}$ (B.), $186^{77}$ (MB:.).

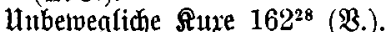

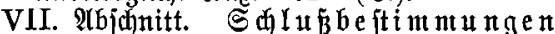
186 ( $\mathfrak{M B S}$.).

Beenoigung ber SInlegungąarbeiten $186^{78}$ (MPB.).

Uebergabe der :lften an ben Sypothefen= beanten $186^{70}$ (MPB.).

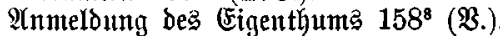

Qumelouny nidjt eingetragener Sied)te $161,162(\mathfrak{B}$.)

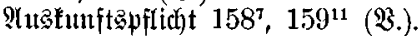

Beijtänoe $158^{10}$ (B.).

Bevolfmächtigte $158^{10}$ (B.).

(Glaubhaftmađung des (sigenthums 10 (B̉.), $159^{12}(\mathfrak{B}$.).

Mehrfadtes (Eigenthunt $160^{19}$ (B.).

Miteigenthum $159^{16}$ (B.).

Bollmadisbeglaubigung $158^{10}$ (:B.), 165 (MPB.).

Boratsję̧ungen Der Bintragung $159^{12}{ }^{14}$ (B.).

$\mathfrak{B i b e r \{ p r i ̈ r l y e} 159^{16}(\mathfrak{B}$.).

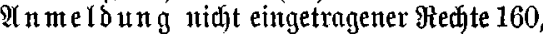
161 (ㄹ.).

Anmeloumgôverfafren.

A. Broed und Gegentand des fahrenz 212-214 (MBB.)

Btwet $212^{1}$ (MPS.).

BSegenftand $212-217^{2},{ }^{3}, 4$ (MPB.).

B. Berfahren 214-227 (MPB.).

I. MMgenteine $\mathfrak{B}$ eftimmungen $212-213$ (MPB.). 3uitündigfeit $214^{5}$ (MOB.).

Iagebud) $214^{6}$ (MVO).

Termingtenlender $214^{\top}$ (MPB.).

Oltenbiloung $214^{8}$ ( $\mathfrak{R B}$.).

Dienftauffidit $215^{\circ}(\mathfrak{M B S}$.)

Bierteljahreaberidhte $215^{10}$ (MPS.).

Bentadyridhtigungen $215^{11}$ ( $\mathfrak{D}:$ B.).

(Sebilfren= 1utb sortofreifeit $215^{12}$ (MBB.).

II. Befundere $\mathfrak{B o r}$ [hriften $216-226$ (MB.)

1. Einleitung bes Berfahrens 216 (MPB.).

Beriduteritattuttg $216^{13}$ (MiB.).

Berfïgung ïber den $\mathfrak{B e g i n n}$ des $\mathfrak{B e r}=$ fabrens $216^{14}$ (MNB.).

2. शufforderung zut श̂neldung 216-219 (MBS.).

Erlaffung ber $\mathfrak{A} u$ fforderung. Snhalt 216, $217^{15}, 16,227$ (MPB.).

Irt ber 'Berïffentlifiung $218^{17}$ (MPB.)

Wiederfolutg ber Einritiunt $218^{18}$ (MBB.).

Ankeftung in bent (Semeinden $218^{10}$ (MQB.).

Dauer der 2 unbeftung $218^{20}$ (MBB.).

Belege für die Beröffentlidung $218^{21}$ (D):B.).

Andere Befanntmad)ungsarten $218^{22}$ (MB.).

(Erjuthen an ben Bitrgermeifter $218^{\mathbf{2 3}}, 228$ ( $\mathfrak{M} \mathfrak{B}$.).

Mittheilungen anßehöroen 21924,237(MPB.)

3. Beurfutudungen 219 (MPB.).

Butitändigleit, Foun $219^{25}$ (MSB.).

4. Eröffnung der Aroronungen bes Geridhts. Form $219^{20}$ (MSB.).

5. Formt der Plnmeloungen und (Erflärungen;

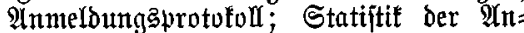
meloungen 219-228 (MPB.).

Frornt ber $\mathfrak{A}$ meloungen und Erflürungen $219^{27}$ (MSB.).

(Sd)riftlidbe $\mathfrak{A}$ nmeloungen und (Erflärungen $220^{28}$ (MiB.).

9lnmeldungstage $220^{29}$ (MPB.)

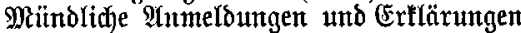
$220^{30}$ (N2R.). 
Plnnteldung sprotofoll $220^{31}$ (MM:B.).

Statifitif ber Fnmeloungen 22032, 231 $(\mathfrak{M} \mathfrak{B}$.).

6. Âmeldung und Eintragung von Mied)tent $221-225$ (MPB.).

Pnmeldungabereditigte $221^{33}$ (9MB.).

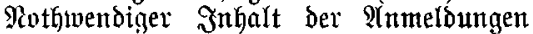
$221^{34}$ ( $\mathfrak{M B}$.).

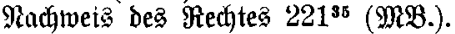

Erfordernif der Butimmunt des $\mathfrak{A n}=$ meloungagegners $222^{3 \theta}$ (MDE.).

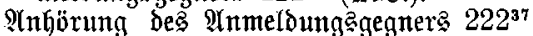
(MiB.).

Ertheilunto ber 3 uftimmung zur cin= tragung $223^{38}$ ( $\mathfrak{M B S}$.).

Berweigerung ber 8uftimmung 223, $224^{30}{ }^{41}$ (MRB.).

Beitpunft der Befolutiffajiung und desิ Bollzuga 224:2 (MRB.)

Stelle, Form und §nbalt der (Fintragung $224^{43}$ (MPB.).

Benadjriditigung von ber fintragung $224^{44}$ (MDB.).

Bebandlung ber vorgelegten urfunden $225^{45}, 46,231$ (MR.).

7. Inmeloung uno Cintragung ber $\mathfrak{B e r}=$

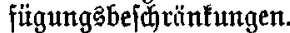

Altwendung ber für bie Ânmeldung von

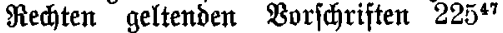
(MiB.).

8. Anmelbung und Eintragung bon ßrote= itationen (Berwabungen, $\mathfrak{B}$ ideripritide). Berfahren $225^{48}$ (MPB.).

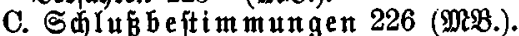

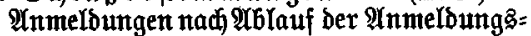
frift $226^{40}$ ( $M P Q$.).

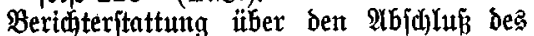
Anmeloutgşoerfahrens $226^{\text {s0 }}$ (MDB.).

P(nlagen 227-231 (MPB).

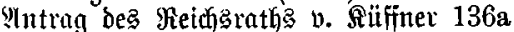

शluffebung eines Folium? 9 ( $\mathfrak{B}$.).

\section{B.}

Begriindung des Enttwurfes 5-16.

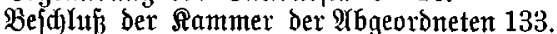

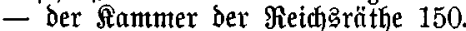

Befibueränderungen, Beritündigund Der

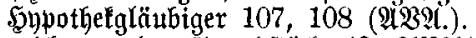

Bezeidnumg ber (śrundititife 42 (92B?.).

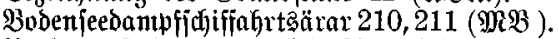

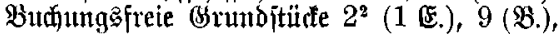

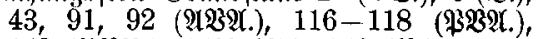

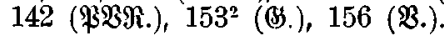

\section{c.}

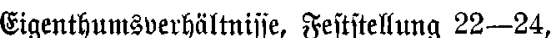
$26-29,32-37,74,75,80-86,88,89$

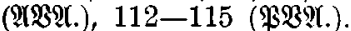

Eintragabewilfigutgen, Beurfumbung $4^{10}$

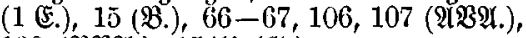
130 (\$2QA.), $154^{10}$ (\&.).

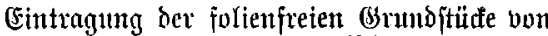
?Intestuegen $2^{1}$ (1 (E.), 9 (B.), 35, 41-43,

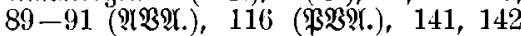
$(\mathfrak{B Q R}), \quad 153^{1}$ (\$3.), $157-160(\mathfrak{B}),$.

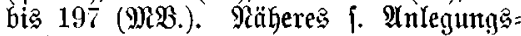
verfahtent.

-., Porau fesungen 10 (⿱⺈.).

Eijenbaftütrat 210 (MRB.).

Entwurf 2-5.

- ber $\mathfrak{A n}$ legungsverotbnung $45-47$.

(Erklärung de bud $161,162(\mathfrak{B}$.$) .$

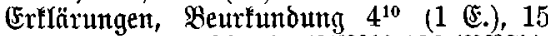

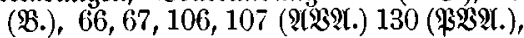
$154^{10}$ (अ.).

F.

Formelle Befandiung des Beję̧entwutrfes

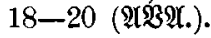

(5).

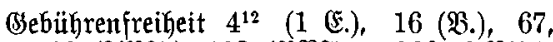

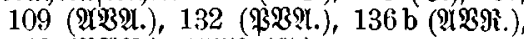
148 (\$YR.), $155^{12}$ (\$S.).

Befithrenitundouttg bis nadh llmidjreifunt $4^{13}(1$ (․) $) 16$ (B.) $), 25,26,67-70,109$,

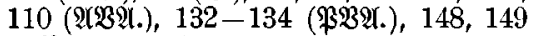
(BRBR.), $155^{13}$ (BS.).

Bemeindebehörden, Mitwirkung im Ein= tragutngaverfahren $2^{3}$ ( 1 (E).), 10 (B). ), 25, $26,30,33,34,35,37,44,76,93,94$

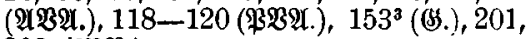
$202(\mathfrak{M} B$.)

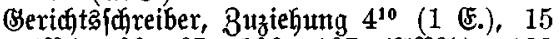

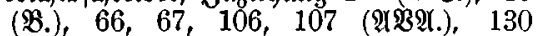
(\$29.), $155^{10}$ (\$).

(BrundfteuerEatafter, Beridftigung 203 (MB .).

Bejammtbejd)

BSefanmthyouthefen 3,4 (1 biz 15 (3.), 22, 25, 28, 48-60,81, 82 ,

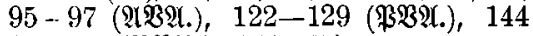
bi 147 (BBR.), 154 (\$.).

- Sefriediaungerredit $3^{5}$ (1 E.), 13 (B).),

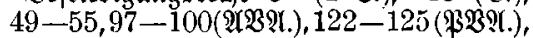
144 (\$BRR.), $154^{5}$ (\$5).

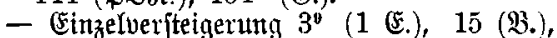

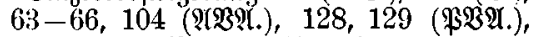
146,147 ( $\mathfrak{3 \Re}$.), $154^{4}$ (\$).).

- Bitszzertriinmerung $3^{\theta}$ (1 (5.), $12-14$

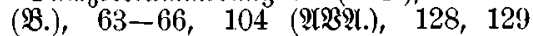

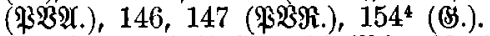

- Realredfle $3^{8}$ (1 (E.), 14 (8.), 63, 104

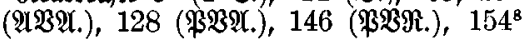
(अ.).

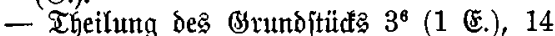

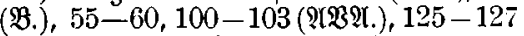

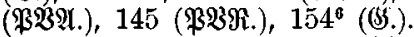

- Iremmiticte $3^{8}(1$ (5.), 14 (B). $), 33-66$, $104(\mathfrak{A B Q}$.) $, 128,129$ (\$3P.), 146, 147

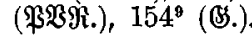


- Uebergangsredt $3^{7}$ (1 (5.), 14 (B.), 60

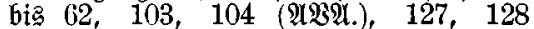

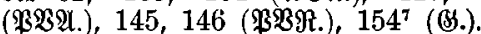

- Beräuperunģรejd)ränfung $3^{8}$ (1 (E.), 14

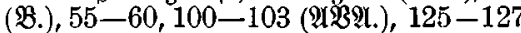

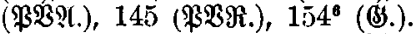

- Bertheilungaverfahren 13 (BB.).

(Sejebestert 153-155.

\section{$\$$.}

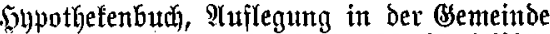
$24,33,36,38,39,40,41,86,87$ (श्YSA).

-, Sejtintmuny 5 (B.).

— Eimriditumg $5^{14}(1$ (E.), 16 (B.) , 70, 71,

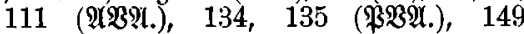

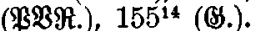

Fnpothefentüger, (Einridutung 204-208 (MBS.).

5nyothefenbuc umo §atajter, Heberein= ftimmung $22,23,24,26,28,29,32,33$, $35,36,37,74,75,80,81,82-86$ (

- unterfdied vom (Srumbhuda 7,8 (B.),

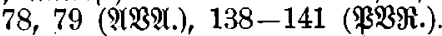

Sinpothefenbudbezirfe 204 (MPB.).

Şupothefentgejes, Beltung für $\mathfrak{A n t a g e v e r =}$ faffren 42 (भis:.).

\section{R.}

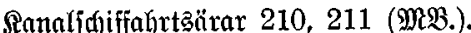
Roftenfreibeit Der Berfandolungen 10 (B.).

\section{$\mathfrak{M R}$.}

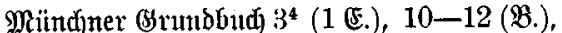
$24,25,27,28,48,94,95$ (2YBM.), 120

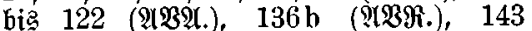

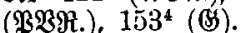

- Ewiggelder $3^{4}(1$ \&.), 11, 12 (B.), 95

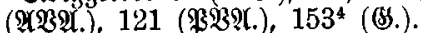

- Brundoienjtbarfeiten $3^{4}(1$ E.), 11 (B.),

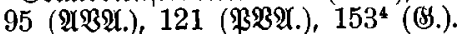

- Bereinigung mit Sypothetenfud) 11,12

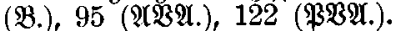

- Bulfifige Eintragungen $3^{4}$ (1 E.), 95

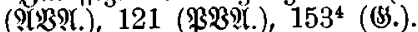

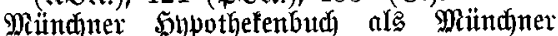
(brunbbudh, f. bie\}e?

$\Re$.

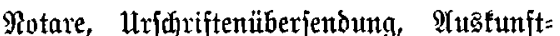

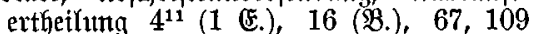

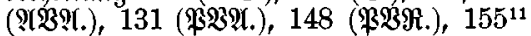
(B).).

Rotariatumfunden, vollzieffbar gewordene, Ueberjendung 164 (Mib.).
D.

Deffentlid)teitsprimzip 5 (B).), 32，36，37, 78,79 .

Drt ber (Serid)tähandlungen 10 (B.).

\$.

ßerjonenregifter 209, 210 (MEB.).

Bfandbudjinftem, Hebergang zum (Sinund=

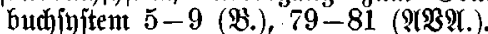

Bfandoerbandentlaffung, Reuntlage eines Foliume 9 (

Blenarverhandolumgen ber Rammer Der $\mathfrak{A} 6$ georoneten $112-136,151$

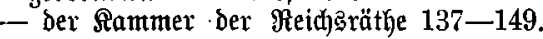

R.

Rethtofilfe $4^{10}(1$ (E.), 15 (B).), 66, 67, 106

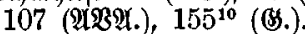

Rechtôprnftifanten, Beurfundung $4^{10}$ (1 E.),

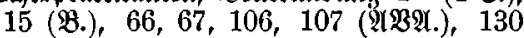
(\$BQ.), $155^{10}$ (\$.).

ธ.

Sadjregifter 163, 164 (MBR.), 209, 210 $(\mathfrak{M}$ B.).

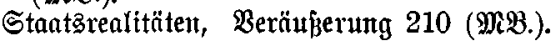

$\mathfrak{B}$.

Berfafren bei der Fintragung folienfreier Brunditiife $2^{3}(1 \quad$ (5.), 9,10 (B.), 44,

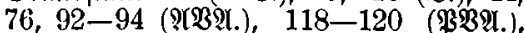

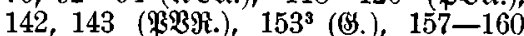

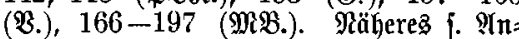
legungaberfahren.

Berfandlungen des STusfintuffes ber Sanmer ber arfgenroneten 17-111.

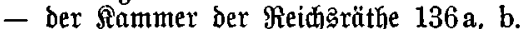

Bermejiungąhehörben (Beometer), Stellung zum (Srumbbud 27, 30, 34, 115 (929.).

Bernehmung ber Betheiligten 10 (B.).

Berträge, Beurfundung $4^{10}(1$ (E.), 15 (B). $)$,

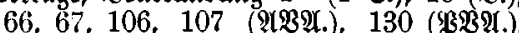
$154^{10}$ (Bs.)

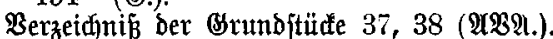
Sollmadten ber Bitrgermeifter 10 (方.).

Borbemerfura 1.

Borlage des Entwurfez 1, 2.

\section{3.}

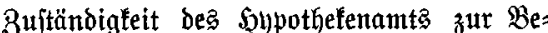
unfunoung $4^{10}$ (1).

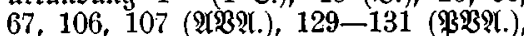

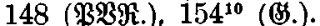

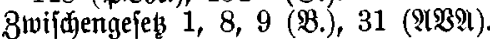

